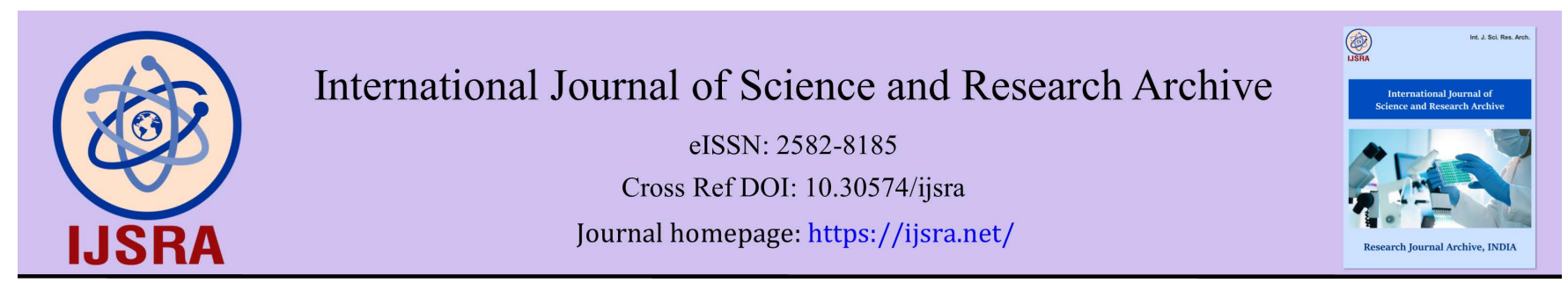

(RESEARCH ARTICLE)

Check for updates

\title{
Anemia during pregnancy: Epidemiological, clinical and therapeutic aspects at the obstetric gynecology department of Labe Regional Hospital, Guinee
}

Boubacar Siddi Diallo 1, ${ }^{*}$, Boubacar Alpha Diallo 1, Aguibou Barry 1, Fatoumata Binta Sow 1, Oumar Diawara ${ }^{1}$, Daniel Leno ${ }^{1}$, Abdourahamane Diallo ${ }^{2}$, Ibrahima Sory Balde ${ }^{2}$, Telly Sy ${ }^{2}$, Yolande Hyjazi ${ }^{1}$ and Namory Keita ${ }^{1}$

${ }^{1}$ University Department of Gynecology Obstetrics, Donka national Hospital, Conakry Guinea.

2 University Department of Gynecology Obstetrics, Ignace Deen national Hospital, Conakry Guinea.

International Journal of Science and Research Archive, 2021, 03(02), 001-005

Publication history: Received on 13 July 2021; revised on 24 August 2021; accepted on 26 August 2021

Article DOI: https://doi.org/10.30574/ijsra.2021.3.2.0121

\begin{abstract}
Objectives: Calculate the frequency of anemia during pregnancy, describe the epidemiological profile and describe the management of anemia during pregnancy in the gynecology-obstetrics Department of the Labé regional hospital.

Methodology: This was a prospective, descriptive study lasting six (6) months from September 1, 2020 to February 28, 2021 All pregnant women who developed anemia and treated were considered for the study in the service. All pregnant women without anemia were included. Our data were analyzed by Epi-Info version 7.2.2.6 software, entered and presented by Pack office 2016 software.
\end{abstract}

Results: The frequency of anemia in pregnant women was $27.61 \%$ in the department. The epidemiological profile of anemia in pregnant women at the Labé regional hospital was that of: a woman aged 25-29 (30.63\%), housewives (72.97\%), women not attending school (66, $67 \%$.), those Coming from the rural areas $(76.58 \%)$, married women $(100 \%)$, pauciparous $(29.73 \%)$ and only $14.42 \%$ did not carry out an antenatal consultation. The anemia occurred in the $3^{\text {rd }}$ trimester $(76.58 \%)$. The main reason for consultation was paleness of the conjunctivae and integuments $(92.79 \%)$ and malaria the dominant antecedent (60.36\%). Severe anemia was the most dominant clinical form (54.96\%). Transfusion was performed in $54.95 \%$ of women with anemia. The average length of stay was 4.9 days with extremes of 1 and 10 days. The maternal prognosis was favorable in $96.40 \%$ with a maternal death rate of $3.60 \%$.

Conclusion: Anemia is a major problem for pregnant women in the Gynecology-Obstetrics department at the Labé regional hospital. The improvement of the maternal prognosis would pass by chemoprophylaxis of malaria, the use of impregnated mosquito nets with long-lasting insecticide (LLINs), the prevention of intestinal parasitoses, the regular administration of iron, folic acid and the provision of quality prenatal consultation.

Keywords: Anemia; Pregnancy; Labé; Obstetric gynecology

\section{Introduction}

The definition of anemia in pregnant women varies depending on the trimester of pregnancy and is based on a hemoglobin level of less than $11 \mathrm{~g} / \mathrm{dL}$ in the first and third trimesters and less than $10.5 \mathrm{~g} / \mathrm{dL}$ in the second trimester. This decrease in hemoglobin level during pregnancy is due to physiological hemodilution [1]. It is a decrease in the level of functional hemoglobin below the normal values which according to the World Health Organization (WHO) are of: 13g / $\mathrm{dl}$ in men, $12 \mathrm{~g} \mathrm{/} \mathrm{dl}$ in non-pregnant women and $11 \mathrm{~g} \mathrm{/} \mathrm{dl}$ in pregnant women [2]. Anemia is the most common public health problem encountered in the world in general, and in developing countries in particular [3]. These consequences

\footnotetext{
${ }^{*}$ Corresponding author: Boubacar Siddi Diallo

University Department of Gynecology Obstetrics, Donka national Hospital, Conakry Guinea.
} 
can be felt at any level: maternal asthenia, premature delivery, restriction of growth in utero, overexposure to infections, fatigue and frailty in the postpartum period with possible impact on the postpartum. establishment of the mother-child link [4]. In view of all these consequences and the multiple possible etiologies, the WHO recommends, in pregnant women, preventive measures to systematically and concomitantly fight against iron deficiency and itamines (systematic iron and folate supplementation throughout pregnancy) [5]. According to recent data from the World Health Organization (WHO), more than 2 billion people or $40 \%$ of the world population suffer from anemia. The most affected populations are pregnant women (50\%); women of childbearing age (35\%) [6].

In the United States, according to (the National Health and Nutrition Examination Survey), 5 to $10 \%$ of women aged 20 to 44 have iron deficiency. And in a prospective study conducted in Camden (New Jersey, USA), 28\% of pregnant women were anemic, but only 3.8\% met the definition of iron deficiency anemia, although nearly $10 \%$ had reservations. low iron [7]. In Morocco: Sellam, EB, et al. in 2014 reported in a cross-sectional study $37.2 \%$ of iron deficiency anemia in pregnant women and 32.6\% in women of childbearing age [8]. In Cameroon, Charlotte N. et al. in 2016 found in a crosssectional study 39.8\% anemia in pregnant women [9]. In West Africa, Burkina Faso, Nikiema L., et al. reported in a crosssectional study $66 \%$ of anemia in pregnant women [10]. In Guinea, DABO T. in 2016 identified 34.15\% of anemia in pregnant women, at the regional hospital of Mamou [11].

The objectives of this study were to calculate the frequency of anemia during pregnancy, describe the epidemiological profile and describe the management of anemia during pregnancy in the gynecology-obstetrics Department of the regional hospital of Labé

\section{Methodology}

This was a prospective descriptive study lasting six (6) months from September 1, 2020 to February 28, 2021 carried out at the Gynecology-Obstetrics Department of the regional hospital of Labé which is a structure of $2^{\text {nd }}$ level reference in Guinea. All pregnant women who developed anemia and were treated during the study in the ward were included. All pregnant women without anemia were not included. Our data were analyzed by Epi-Info version 7.2.2.6 software, entered and presented by Pack office 2016 software.

The study looked at a continuous series of 111 anemic pregnant women. We carried out an exhaustive recruitment of pregnant women according to the criteria defined above. The variables studied were: epidemiological (frequency, maternal age, occupation, level of education and marital status) obstetric (parity, gestational age, number of prenatal consultations, reasons for consultation, medical history, causes of anemia, degree of anemia, treatment received, duration of hospitalization and maternal prognosis).

\section{Results}

\subsection{Frequency}

The frequency of anemia in pregnant women was $27.61 \%$ in the obstetric gynecology department of the Labé regional hospital.

\subsection{The epidemiological profile}

- Maternal age: the 25-29 age group was the most affected (30.63\%), followed by that of 20-24 years (23, $42 \%)$. The average age was 28.24 with extremes of 16 and 45.

- Occupation: Housewives were the most concerned (72.97\%).

- Level of education: pregnant mothers who were not schooled were the most exposed (66.67\%).

- Marital status: all of our pregnant women were married (100\%).

\subsection{Obstetrics}

- Parity: all parity brackets were affected by anemia with a clear predominance of pauciparas $29.73 \%$, followed by multiparas with $27.93 \%$.

- Gestational age: there is a predominance of anemia during the $3^{\text {rd }}$ trimester $76.58 \%$, followed by the second trimester with $20.72 \%$.

- Number of prenatal consultations: among our pregnant women, $14.42 \%$ had not performed an antenatal consultation. 
- Reasons for consultation: The main reason for consultation was paleness of the conjunctivae and integuments with $92.79 \%$, followed by physical asthenia and dizziness with $87.38 \%$ and $84.68 \%$ respectively.

- History: The medical history is dominated by malaria $72.07 \%$, followed by anemia $18.01 \%$ and intestinal parasitosis $17.11 \%$.

- Causes of anemia: Malaria was the most noted etiology $60.36 \%$, followed by sickle cell anemia $11.71 \%$ and intestinal parasitosis $10.81 \%$.

- Degree of anemia: severe anemia was the most observed clinical form $54.96 \%$. It was moderate in $24.32 \%$ and $20.72 \%$ of patients had mild anemia.

- Duration of hospitalization: we found that $49.55 \%$ of patients were hospitalized between 4 and 8 days. The average length of stay was 4.9 days with extremes of 1 and 10 days.

- Treatment received: in our series, 54.95\% of our pregnant women were transfused. Iron and folic acid were administered as a curative treatment in $37.84 \%$ of our pregnant women.

- Maternal prognosis: it was favorable in $96.40 \%$ against $3.60 \%$. Of registered maternal deaths.

\section{Discussion}

\subsection{Frequency}

The frequency of anemia in pregnant women was $27.61 \%$ in the obstetric gynecology department of the regional hospital of Labé. This result is lower than those reported by Nadia O. et al. [12] in Morocco and ADEBO A.A et al. [13] in Benin who found respectively $41 \%$ and $38.39 \%$ anemia during pregnancy.

\subsection{The epidemiological profile}

- Maternal age: the 25-29 age group was the most affected (30.63\%), followed by that of 20-24 years (23, 42\%). The average age was 28.24 with extremes of 16 and 45 . Our result is superimposable on the result found by DABO T. [11], i.e an average age of 26.25 years with extremes of 15 and 42 years. The most affected age group is 15-19 years (27.38\%). ADEBO A A. et al. [14] in 2019 in Benin found a similar result, an average age of 27.74 \pm 5.51 years. The most affected age group is that of 18 to 35 years (85\%). This could be explained by the fact that this age group represents the population where the procreation rate is the highest in our socio-economic and cultural context. -

- Occupation: Housewives were the most affected (72.97\%). This rate is comparable to that of NIKIEMA L. et Coll. [10] who reported $78.60 \%$ of housewives against $21.1 \%$ of employees. This observation could be the fact that housewives are numerous in the region of Labé.

- Level of education: pregnant women who did not attend school were the most exposed (66.67\%). Our result corroborates that of KEITA M. [15] who reported $84.74 \%$ of anemia in pregnant women who did not attend school. According to ADEBO A A. et al. [14], the level of education is a factor significantly associated with anemia during pregnancy. This could be explained by the fact that a mother who does not have an adequate level of education will find it difficult to comply with medical prescriptions and recommendations.

- Marital status: all of our pregnant women were married (100\%) This result is identical to that reported by Nadia 0. et al. [12] in Morocco, who reported that all anemic women who participated in their study at the time of the survey were married, but higher than the $80.7 \%$ reported by Charlotte N.T et al. [9] in Cameroon.

\subsection{Obstetrics}

- Parity: all parity extremes were affected by anemia. This result is comparable to those of NIKIEMA L. et al. [10] in Burkina Faso who found a predominance in pauciparas with 41\%, unlike those of DABO T. [11] who reported a predominance of nulliparas and paucipares with respective rates of $32.14 \%$ and $26,20 \%$ of cases. According to Demmouche A. et al. [16] closely spaced pregnancies are a risk factor for anemia during pregnancy.

- Gestational age: there is a predominance of anemia during the $3^{\text {rd }}$ trimester $76.58 \%$, followed by the second trimester with $20.72 \%$. This result is comparable to those found by Florent D.J. et al. [17] in Cameroon, ie $70.88 \%$ in the third quarter and $29.12 \%$ in the second quarter. Our study shows that the anemia is higher the older the pregnancy is. This could be explained by the fact that the iron requirements are very high in the last trimester of pregnancy not only for the formation of the placental mass, but also for the development of the fetus.

- Number of prenatal consultations: among our pregnant women, $14.42 \%$ had not performed an antenatal consultation. KEITA M. [15] at the prefectural hospital of Siguiri and Dramane I.K [18] in Mali reported 72.88\% and $67.9 \%$, respectively, of patients who did not have an antenatal consultation. This could be explained by the 
lack of awareness of the importance of antenatal consultation in rural areas and the distance from health structures.

- Reasons for consultation: The main reason for consultation was paleness of the conjunctivae and integuments with $92.79 \%$, followed by physical asthenia and dizziness with $87.38 \%$ and $84.68 \%$ respectively. Our result is different from that by DABO T. [11] who reported that anorexia, physical asthenia, paleness of the conjunctiva and dyspnea were the main symptoms with $96.43 \%$ respectively; $88.10 \% ; 86.90 \%$ and $69.04 \%$ of cases.

- Causes of anemia: Malaria was the most noted etiology $60.36 \%$, followed by sickle cell anemia $11.71 \%$ and intestinal parasitosis 10.81\%. Our result is lower than that of DABO T. [11] and KEITA M. [15] who reported $76.19 \%$ and $84.74 \%$, respectively, of malaria. This could be explained by the non-compliance with intermittent preventive treatment.

- Degree of anemia: severe anemia was the most observed clinical form $54.96 \%$. It was moderate in $24.32 \%$ and $20.72 \%$ of patients had mild anemia. Our result is lower than that reported by KEITA M. [15] who found 75.42\% severe anemia but contrary to those of Nadia 0. et al. [12] in Morocco, and Harir N. [19] in Algeria who reported that the most frequent clinical form was mild anemia followed by moderate anemia with respectively, $56.6 \%, 40.6 \%$ and $67.04 \%, 31.81 \%$ of cases. This could be explained by the absence of systematic iron supplementation.

- Duration of hospitalization: we found that $49.55 \%$ of patients were hospitalized between 4 and 8 days. The average length of stay was 4.9 days with extremes of 1 and 10 days. Our result is superimposed on that found by Dramane I.K [18] in Mali, ie 48.3\% of pregnant women hospitalized for anemia between 4 and 8 days.

- Treatment received: in our series, $54.95 \%$ of our pregnant women were transfused.

This result is higher than that of DABO T. [11], but lower than that of KEITA M. [15] who reported 46.43\% and 75.42\% of pregnant women transfused, respectively. The transfusion is made on the basis of the clinical tolerance of the anemia and the hemoglobin level. Thus a hemoglobin level of less than $8 \mathrm{~g} / \mathrm{dl}$ even with good tolerance of anemia, pregnant women must be transfused in order to ensure sufficient nutritional intake for the fetus and to prevent complications.

Iron and folic acid in curative treatment concerned $37.84 \%$ of our pregnant women. This treatment concerned anemic pregnant women whose clinical condition did not require immediate blood transfusion.

- Maternal prognosis: it was favorable in $96.40 \%$ against 3.60\%. Of recorded maternal deaths. Our result is identical to those of Dramane I.K [18] in Mali, who in his series reported $96.40 \%$ favorable outcome and 3.6\% deaths. This could be explained by the delay in evacuation on one hand and by the temporary unavailability of compatible blood in the blood bank on the other hand.

\section{Conclusion}

Anemia is a major problem for pregnant women in the Gynecology-Obstetrics Department of the Labé Regional Hospital. The improvement of the maternal prognosis would pass by chemoprophylaxis of malaria, the use of impregnated mosquito nets with long-lasting insecticide (LLINs), the prevention of intestinal parasitoses, the regular administration of iron, folic acid and the provision of quality prenatal consultation.

\section{Compliance with ethical standards}

\section{Disclosure of conflict of interest}

No conflict of interest.

Statement of informed consent

Informed consent was obtained from all individual participants included in the study.

\section{References}

[1] Caroff-Petillon A. statement of anemia screening during pregnancy, retrospective study carried out at the Best University Hospital Center. La Revue Sage-femme. 2008; 7: 51-55. 
[2] Sagara S. Epidemiological and prognostic study of anemia in pregnant women at the Koro reference health center, Thesis Med Mali. 2019; P7.

[3] Dogoni L. Epidemiological, clinical and therapeutic study of anemia in pregnancy at the Kadiolo CS Ref, Thesis Med. Mali. 2014; P18.

[4] Rotoba Z. Biological diagnosis of anemia in pregnant women, Doctorat en Med, Morocco. 2014.

[5] Koura KG, Briand V, Massougbodji A, Chippau JP, Cot M, Garcia A. Prevalence and etiology of anemia in pregnant women in southern Benin at the time of the change in national policy of taking charged. Med Trop. 2011; 71: 6367.

[6] Ali Z, Hicham E, Karim Mohamed M, Mohamed E. Etiological profile of anemia in an internal medicine department Pan African Medical Journal. 2017; 26: 10.

[7] Legroux M. Screening and management of anemia in low-risk pregnancies, Diplôme d'état de Sage-femme, France. 2010; P7.

[8] Sellam EB, Bour A. Anemia in women of childbearing age in Morocco (Oujda-Angad Prefecture), 2014 ; 32 : 35-44.

[9] Charlotte NT, Eveline Ngouadjeu DT, Arlette GN, Théophile Nana N, Gregory Halle E, Eugene Belley P. Prevalence and factors associated with anemia in pregnancy at the Douala General Hospital. Pan African Medical Journal. 2016; 25: 133.

[10] Nikiema L, Vocouma A, Sondo B, Martin-Prevel Y. Nutritional determinants of anemia in pregnant women and after pregnancy in urban settings in Burkina Faso; January-December 2010, Science and technology, Health science. $2010 ; 33, \mathrm{~N}^{\circ} 1$ and 2 .

[11] Dabo T. Anemia and Pregnancy: Epidemiological, clinical and therapeutic aspects at the Mamou Regional Hospital. Thesis Med Guinea. 2016; N² 261.

[12] Nadia 0, Kabira T. Ghizlane B, Ezzahra Jarhmouti F, Abdellatif B. Prevalence and social determinants of anemia among pregnant women in the province of Essaouira, Morocco. public-health-review. 2018; 5 (30): $735-745$.

[13] Adebo AA, Yessoufou AG, Kpanou CM, Bouko MB, Soumanou MM, Sezan A. Anemia and associated factors in pregnant women seen in consultation at the Ekpè Health Center (South of Benin). 2018; 40: 35-41.

[14] Adebo AA, Yessoufou AG, Alamou OF, Bio Bouko M, Soumanou MM, Sezan A. Epidemiological aspects of anemia in pregnant women seen in consultation at the university hospital of abomey-calavi / so-ava ( south benin). 2019.

[15] Keita M. Anemia and pregnancy: Epidemiological-clinical aspect and treatment at the prefectural hospital of Siguiri. Thesis Med Guinea. $2017 \mathrm{~N}^{\circ} 0148$.

[16] Demmouche A, Moulessehoul S. Prevalence of iron deficiency anemia during pregnancy in the wilaya of Sidi Bel Abbes (western Algeria). Antropo. 2010; 21: 39-48.

[17] Florent DJ, Thierry Franck NH, Noel N, Noubom M, Fomulu N. Prevalence of iron deficiency anemia among pregnant women in Yaoundé. Health Sci. Dis. 2016; 17(1).

[18] Dramane IK. Epidemiological clinical and therapeutic study of severe anemia in pregnancy at Sikasso hospital. Thesis Med Mali. 2009.

[19] Harir N, Zeggai S, Drider ZH, Belkacem A. Nutritional intake of anemic pregnant women in the first trimester of pregnancy. Algeria. 2015; 34: 45-53. 